\title{
SOME LOCAL PROPERTIES OF THE SOLUTIONS OF SECOND-ORDER DIFFERENTIAL EQUATIONS
}

\author{
JIUYI CHENG and JOHN ROSSI
}

(Received 8 March 1993)

Communicated by P. C. Fenton

\begin{abstract}
We investigate the asymptotics and zero distribution of solutions of $w^{\prime \prime}+A w=0$ where $A$ is an entire function of very slow growth. The results parallel the classical case when $A$ is assumed to be a polynomial. 1991 Mathematics subject classification (Amer. Math. Soc.): 30D35, 34A20.
\end{abstract}

\section{Introduction}

The second-order differential equation

$$
w^{\prime \prime}+A(z) w=0,
$$

where $A$ is a polynomial, has been studied extensively. A summary of some classical results regarding the asymptotics and zero distribution of solutions to (1.1) is contained in the following theorem (see [2] or [3, Chap.7]).

THEOREM A Let $A(z)=a_{n} z^{n}+\cdots+a_{0}$ be a polynomial, $a_{n} \neq 0$ and $\alpha_{n}=\arg a_{n}$. For given $\epsilon>0$ define $\theta_{k}=\left(-\alpha_{n}+2 k \pi\right) /(n+2), k=0, \ldots, n+1, S_{k}{ }^{+}(\epsilon)=\{z$ : $\left.\theta_{k}+\epsilon \leq \arg z \leq \theta_{k+1}\right\}, S_{k}{ }^{-}(\epsilon)=\left\{z: \theta_{k} \leq \arg z \leq \theta_{k+1}-\epsilon\right\}, S_{k}(\epsilon)=S_{k}{ }^{+}(\epsilon) \cap S_{k}{ }^{-}(\epsilon)$ and $S_{k}=S_{k}{ }^{+}(\epsilon) \cup S_{k}{ }^{-}(\epsilon)$. Then there exist $n+2$ solutions $g_{k}, k=0, \ldots, n+1$, of (1.1) analytic in $S_{k-1}{ }^{+}(\epsilon) \cup S_{k} \cup S_{k+1}{ }^{-}(\epsilon)$ with $g_{k-1}$ and $g_{k}$ linearly independent and for all $z \in S_{k-1}{ }^{+}(\epsilon) \cup S_{k} \cup S_{k+1}{ }^{-}(\epsilon)$

$$
\text { (1.2) } g_{k}(z)=(1+o(1))\left(a_{n} z^{n}\right)^{-1 / 4} \exp \left\{\frac{2}{n+2} \sqrt{\left|a_{n}\right|} e^{i \alpha_{n} / 2}(-1)^{k} i z^{(n+2) / 2}(1+o(1))\right\}
$$

as $|z| \rightarrow \infty$.

(C) 1995 Australian Mathematical Society 0263-6115/95 \$A2.00+0.00 
For transcendental $A$ not much is known about asymptotic representations of solutions of (1.1) even when the order of $A$ is less than one.

In this note, we consider the equation (1.1) with a transcendental entire function $A(z)$ which grows only slightly faster than a polynomial, namely it satisfies

$$
\varlimsup_{r \rightarrow \infty} \frac{\log \log M(r, A)}{\log \log r}=p<2,
$$

where $M(r, A)=\sup _{|z|=r}|A(z)|$. We describe the solution of (1.1) in a union of a sequence of annuli which is quite large in the sense that the set of moduli of the points in its complement has logarithmic density (see the definition below) zero in $(1, \infty)$. Specifically we obtain a local result similar to (1.2) in Theorem A in the intersections of certain sectors and annuli. The idea can be stated as follows. Under the condition (1.3), a result (see Lemma 2) in Wiman-Valiron theory implies that the entire function $A(z)=\sum_{n=0}^{\infty} a_{n} z^{n}$ is equal to $(1+o(1)) a_{N} z^{N}$ as $|z| \rightarrow \infty$ in the above union of annuli, where $N=N(r)$ is the central index of $A(z)$. Then by use of the Liouville transform (3.5) in each annulus we can use the method of Hille locally on the corresponding equation

$$
W^{\prime \prime}+(1-F(Z)) W=0,
$$

where

$$
F(Z)=\frac{A^{\prime \prime}(z)}{4 A(z)^{2}}-\frac{5 A^{\prime}(z)^{2}}{16 A(z)^{3}}
$$

and

$$
W(Z)=A(z)^{1 / 4} w(z) .
$$

Finally, we transfer the result concerning the solution of (1.4) to that of (1.1), again via the Liouville transform.

To state our theorem, let

$$
A(z)=\sum_{n=0}^{\infty} a_{n} z^{n}
$$

and let $\alpha_{n}=\arg a_{n}, n=0,1, \ldots$ Now for any $n$ define for $k=0,1, \ldots, n+1$

$$
S_{n, k}(\epsilon)=\left\{z: \frac{-\alpha_{n}+2(k-1) \pi}{n+2}+\epsilon<\arg z<\frac{-\alpha_{n}+2(k+2) \pi}{n+2}-\epsilon\right\}
$$

where $\epsilon>0$.

We recall the definition of logarithmic density of a set $E \subset[0, \infty)$ :

$$
\log \operatorname{dens} E=\lim _{r \rightarrow \infty} \frac{\operatorname{l.m} \cdot(E \cap(1, r))}{\log r}
$$


provided that the limit exists. Here 1.m. $(E)=\int_{E \cap(1, \infty)} d r / r$ is the logarithmic measure of $E$.

Throughout the remainder of this paper, we will use the letter $C$ to denote a constant which depends on $A(z)$ (or on a function $F(z)$ which will ultimately depend on $A(z)$ ).

We now state our main result:

THEOREM. Let $A(z)$ be an entire function satisfying (1.3) and of the form (1.7) and let $0<\eta<2-p$. Then there exists an infinite sequence of positive integers $\mathscr{G}$ and two sequences $\left\{r_{n}\right\}_{n \in \mathscr{G}}$ and $\left\{s_{n}\right\}_{n \in \mathscr{G}}$ satisfying $0<r_{n}<s_{n}<r_{m}<s_{m}$, whenever $n<m ; n, m \in \mathscr{G}$, such that the logarithmic density of $\bigcup_{n \in \mathscr{G}}\left(s_{n}, r_{n+1}\right)$ in $(1, \infty)$ is zero. Furthermore for any $\epsilon>0$ and $n \in \mathscr{G}$, the equation (1.1) has $n+2$ pairwise linearly independent solutions $u_{n, k}, k=0,1, \ldots, n+1$, analytic in

$$
\Omega_{n, k}(\epsilon)=\left\{z: r_{n} \leq|z| \leq s_{n}\right\} \cap S_{n, k}(\epsilon)
$$

such that for all $z \in \Omega_{n, k}(\epsilon)$

$$
u_{n, k}(z)=(1+o(1))\left(a_{n} z^{n}\right)^{-1 / 4} \exp \left\{\frac{2}{n+2} \sqrt{\left|a_{n}\right|} e^{i \alpha_{n} / 2}(-1)^{k} i z^{(n+2) / 2}(1+o(1))\right\} .
$$

Moreover, if $u$ is a solution of $(1.1)$, then

$$
u(z)=\alpha_{n, k} u_{n, k}(z)+\beta_{n, k} u_{n+1, k}(z)
$$

and either $\alpha_{n, k} \beta_{n, k}=0$ and $u$ has no zeros in $\Omega_{n, k}(\epsilon) \cap \Omega_{n, k+1}(\epsilon)$ or $\alpha_{n, k} \beta_{n, k} \neq 0$ and the set of all zeros of $u$ in $\Omega_{n, k}(\epsilon) \cap \Omega_{n, k+1}(\epsilon)$ has the form

$$
\left\{\left[\frac{(-1)^{k}(n+2)}{4\left(1+\delta_{n, k, l}\right) \sqrt{\left|a_{n}\right|}} e^{-i \alpha_{n} / 2}\left((2 l+1) \pi+i \gamma_{n, k}+\lambda_{n, k, l}\right)\right]^{2 /(n+2)}: l \in \mathbb{Z}\right\},
$$

where $\gamma_{n, k}=\log \alpha_{n, k} / \beta_{n, k}\left(\arg \alpha_{n, k} / \beta_{n, k} \in[0,2 \pi]\right)$,

$$
\left|\delta_{n, k, I}\right|<\exp \left\{-\frac{1}{2}\left(\log r_{n}\right)^{\eta}\right\}
$$

and

$$
\left|\lambda_{n, k, l}\right|<\frac{C e^{n+2}(n+2)}{\sqrt{\left|a_{n}\right|}}\left(r_{n}\right)^{-(n+2) / 2} \rightarrow 0, \quad n \rightarrow \infty .
$$

The theorem gives very good asymptotics for solutions to (1.1) in the spirit of Theorem A. We emphasize however that our results are purely local. This can be seen best in the result pertaining to the distribution of zeros. Indeed the theorem produces a family of Stoke's rays in each annulus similar to those of Theorem A. The main difference is that the constant $\gamma_{n, k}$ may grow quite rapidly as $n \rightarrow \infty$. (This problem does 
not occur in Theorem A, since there is but one annulus!) Thus closeness to the rays depends unfortunately but at least explicitly on the particular solution. It is fairly certain that any better result would depend on continuing the asymptotics of the $u_{n, k}$ into neighboring annuli. With virtually no information on $A(z)$ in the exceptional set of logarithmic density zero, this strikes the authors as a formidable task. It will become clear in the proof that the sequence $\mathscr{G}$ is merely the range of the central index $N(r)$ of $A$ for $r$ sufficiently large.

It is fairly easy to construct examples satisfying the conditions of our Theorem for any $p$ between 1 and 2 . Specifically let $1<k<\infty$ and define $A(z)=$ $\sum_{n=0}^{\infty} z^{n} / \exp n^{k}$. It is also easy to show using the relationship between the maximum modulus of an entire function and the maximum term of its series expansion (see [1]) that $A$ satisfies (1.3) with $p=1+1 /(k-1)$.

We mention in passing that a similar result even with $p=2$ in (1.3) would be much more difficult since the power series in this case is dominated by a polynomial and not a monomial in each annulus and the degree and leading coefficient of this polynomial cannot be precisely determined. However our method does indeed apply to an $A$ of any order provided that its series expansion is sufficiently 'gapped.' Specifically if $A$ is dominated by its maximum term in a sequence of annuli centered at the origin, the ratio of whose outer and inner radii approaches infinity, a version of our theorem still holds. A function with Hadamard gaps provides such an example. We leave the details to the reader.

This paper is part of the first author's PhD dissertation written under the direction of the second author and submitted to Virginia Polytechnic Institute and State University.

\section{Lemmas}

The following lemma summarizes some aspects of the Wiman-Valiron theory which we will need in the sequel. It can be found in [1, Theorem 12].

LEMMA 1. Suppose that $f(z)$ is an entire function and $N(r)$ is the central index of $f(z)$. Let $j$ be a fixed non-negative integer. Then there exists a set $E$ of finite logarithmic measure such that if $r=|z| \notin E$ and $r \rightarrow \infty$ we have

$$
M\left(r, f^{(j)}\right)=(1+o(1))\left(\frac{N(r)}{r}\right)^{j} M(r, f) .
$$

An easy modification of the proof of [1, Lemma 4, p.329] gives the following lemma.

LEMMA 2. Suppose that $A(z)$ is an entire function satisfying (1.3) and of the form (1.7) with central index $N(r)$. Then denoting by $\mathscr{G}$ the range of $N(r)$ there exist two 
sequences $\left\{r_{n}^{\prime}\right\}_{n \in \mathscr{G}}$ and $\left\{s_{n}^{\prime}\right\}_{n \in \mathscr{G}}$ satisfying $0<r_{n}^{\prime}<s_{n}^{\prime}<r_{m}^{\prime}<s_{m}^{\prime}$, whenever $n<m$; $n, m \in \mathscr{G}$ and $0<\eta<2-p$, such that the logarithmic density of $\bigcup_{n \in \mathscr{G}}\left(s_{n}^{\prime}, r_{n+1}^{\prime}\right)$ is zero. Furthermore if $r=|z| \in \bigcup_{n \in \mathscr{G}}\left[r_{n}^{\prime}, s_{n}^{\prime}\right]$ we have

$$
\sum_{j \neq N(r)}\left|a_{j}\right| r^{j}<\left|a_{N(r)}\right| r^{N(r)} \exp \left\{-(\log r)^{\eta}\right\}
$$

where $N(r) \equiv n<(\log r)^{q-1}$ if $r \in\left[r_{n}^{\prime}, s_{n}^{\prime}\right]$ whenever $n \in \mathscr{G}$ and $p<q<2-\eta$.

The following lemma is a local version of Hille's method (see [3, Chap.7]). It is basically due to Langley [4, Lemma 1]. Since our regions differ somewhat from his, we offer a detailed proof.

LEMMA 3. Let $0<\epsilon<1 / 4$ and let $F(Z)$ be an analytic function in $\Omega_{k}^{\prime}$ satisfying $|F(Z)| \leq C /|Z|^{2}$ for all $Z \in \Omega_{k}^{\prime}$, where

$\Omega_{k}^{\prime}=\{Z: \max \{1,10 C\} \leq R \leq|Z| \leq S<\infty,(k-1+\epsilon) \pi \leq \arg Z \leq(k+2-\epsilon) \pi\}$, is a closed region on the Riemann surface of $\log Z$ with $S>R(\sin \pi \epsilon)^{-1}$. Then the equation (1.4) has a solution $U_{k}(Z)$ in

$$
\Omega_{k}=\left\{Z: R(\sin \pi \epsilon)^{-1} \leq|Z| \leq S\right\} \cap \Omega_{k}^{\prime}
$$

such that

$$
U_{k}(Z)=\left(1+\epsilon_{k}(Z)\right) \exp \left\{(-1)^{k} i Z\right\}
$$

in $\Omega_{k}$, where $\epsilon_{k}(Z)$ satisfies $\left|\epsilon_{k}(Z)\right|<C / \epsilon|Z|$ in $\Omega_{k}, k \in Z$.

PROOF. It suffices to prove the lemma when $k=0$ and $k=1$. The general case follows similarly. We first assume that $k=0$. Choose a solution $u$ of the equation

$$
u^{\prime \prime}+2 i u^{\prime}-F u=0
$$

such that $u(X)=1$ and $u^{\prime}(X)=0$ where $X=S e^{\pi i / 2}$. Now set

$$
w(Z)=u(Z)-1+\frac{1}{2 i} \int_{X}^{Z}\left(e^{2 i(t-Z)}-1\right) F(t) u(t) d t,
$$

where the integral is independent of path in the closed region $\Omega_{0}^{\prime}$ on the Riemann surface of $\log Z$, since $F$ is defined and analytic there. Differentiation of (2.2) gives

$$
w^{\prime}(Z)=u^{\prime}(Z)-\int_{X}^{Z} e^{2 i(t-Z)} F(t) u(t) d t
$$

and $w^{\prime \prime}(Z)=-2 i w^{\prime}(Z)$, so that since $w(X)=w^{\prime}(X)=0, w$ vanishes identically on $\Omega_{0}^{\prime}$. Now let $Z \in \Omega_{0}$. Let $(-1+\epsilon) \pi \leq \arg Z<-\pi / 2$. Then noting that 
$S>R(\sin \pi \epsilon)^{-1}$, we choose the path $\Gamma$ of integration in $\Omega_{0}^{\prime}$ to be the vertical line-segment from $X$ to $X^{\prime}=(-|Z| \sin (\arg Z)) e^{\pi i / 2}$, followed by the half circle $|t|=-|Z| \sin (\arg Z)$ from $X^{\prime}$ to $X^{\prime \prime}=(-|Z| \sin (\arg Z)) e^{-\pi i / 2}$ in the right half-plane, and then followed by the horizontal line-segment from $X^{\prime \prime}$ to $Z$. Since $\operatorname{Im}(t-Z) \geq 0$ for $t \in \Gamma$, if $d \tau$ denotes the arc-length on $\Gamma,(2.2)$ and the fact that $w$ is identically 0 , give

$$
|u(Z)-1| \leq \int_{X}^{Z}|F(t) u(t)| d \tau
$$

Set

$$
W(\zeta)=\log \left(1+\int_{X}^{\zeta}|F(t) u(t)| d \tau\right)
$$

where $\zeta \in \Gamma$. Then by (2.3), $\frac{d W}{d \zeta} \leq|F(\zeta)|$. So

$$
\begin{aligned}
W(Z) \leq & W(X)+\int_{X}^{Z}|F(t)| d \tau \\
= & \int_{X}^{X^{\prime}}|F(t)| d \tau+\int_{X^{\prime}}^{X^{\prime \prime}}|F(t)| d \tau+\int_{X^{\prime \prime}}^{Z}|F(t)| d \tau \\
\leq & \int_{-|Z| \sin (\arg Z)}^{S} \frac{C}{y^{2}} d y+\frac{C}{|Z|^{2} \sin ^{2}(\arg Z)} \cdot \pi(-|Z| \sin (\arg Z)) \\
& +\int_{0}^{-|Z| \cos (\arg Z)} \frac{C}{x^{2}+|Z|^{2} \sin ^{2}(\arg Z)} d x \\
\leq & C\left(-\frac{1}{|Z| \sin (\arg Z)}-\frac{1}{S}\right)-\frac{\pi C}{|Z| \sin (\arg Z)}-\frac{C \cos (\arg Z)}{|Z| \sin (\arg Z)} \\
< & C /|Z| \sin \pi \epsilon .
\end{aligned}
$$

Since $0<\epsilon<1 / 4$, we have $\sin \pi \epsilon>\pi \epsilon / 2$, and hence

$$
W(Z)<C / \epsilon|Z|
$$

Thus (2.3) implies that

$$
|u(Z)-1| \leq \exp (C / \epsilon|Z|)-1 \leq C / \epsilon|Z| .
$$

Now set $U_{0}(Z)=e^{i Z} u(Z)$; then $U_{0}(Z)$ solves (1.4), and (2.1) (when $k=0$ ) follows at once. If $3 \pi / 2<\arg Z \leq(2-\epsilon) \pi$, we choose a similar path of integration in $\Omega_{0}^{\prime}$, but now the half circle is in the left half-plane. If $-\pi / 2 \leq \arg Z \leq 3 \pi / 2$, we just 
choose the path of integration in $\Omega_{0}$ to be the vertical line-segment from $X$ to $|Z| e^{\pi i / 2}$, followed by the arc of the circle $|t|=|Z|$ from $|Z| e^{\pi i / 2}$ to $Z$. Then

$$
\begin{aligned}
W(Z) & \leq \int_{X}^{|Z| e^{\pi / 2}}|F(t)| d \tau+\int_{|Z| e^{\pi / 2}}^{Z}|F(t)| d \tau \\
& \leq \int_{|Z|}^{S} \frac{C}{y^{2}} d y+\frac{C}{|Z|^{2}} \cdot \pi|Z| \leq C /|Z|,
\end{aligned}
$$

and (2.1) (when $k=0$ ) follows at once.

To prove the lemma in the case $k=1$, choose a solution $v$ of

$$
v^{\prime \prime}-2 i v^{\prime}-F v=0
$$

such that $v(Y)=1$ and $v^{\prime}(Y)=0$ where $Y=S e^{3 \pi i / 2}$. The integral equation for $v$ is

$$
v(Z)-1=\frac{1}{2 i} \int_{Y}^{Z}\left(e^{-2 i(t-Z)}-1\right) F(t) v(t) d t
$$

and we choose a path of integration on which $\operatorname{Im}(t-Z) \leq 0$. Finally we set $U_{1}(Z)=e^{-i Z} v(Z)$.

LEMMA 4. Let $C, \epsilon, F, \Omega_{k}^{\prime}, \Omega_{k}$ and $U_{k}$ satisfy all the conditions in Lemma 3 and let $R \geq 4 \sqrt{2} \pi C$. Suppose that $U=\alpha_{k} U_{k}+\beta_{k} U_{k+1}$ is a solution of (1.4) in

$$
\Omega=\left\{Z: R(\sin \pi \epsilon)^{-1} \leq|Z| \leq S<\infty,-\infty<\arg Z<\infty\right\},
$$

a closed region on the Riemann surface of $\log Z$. Then either $\alpha_{k} \beta_{k}=0$ and $U$ has no zeros in $\Omega_{k} \cap \Omega_{k+1}$ or $\alpha_{k} \beta_{k} \neq 0$ and the set of all zeros of $U$ in $\Omega_{k} \cap \Omega_{k+1}$ is of the form

$$
\left\{(-1)^{k}\left[(2 l+1) \pi+i \gamma_{k}+\lambda_{k, l}\right] / 2: l \in \mathbb{Z}\right\},
$$

where $\gamma_{k}=\log \left(\alpha_{k} / \beta_{k}\right) \quad\left(\arg \left(\alpha_{k} / \beta_{k}\right) \in[0,2 \pi]\right)$ and $\lambda_{k, l} \in \mathbb{C}$ satisfies

$$
\left|\lambda_{k, l}\right|<C / R \text {. }
$$

PROOF. By Lemma 3, the equation (1.4) has two linearly independent solutions $U_{k}$ and $U_{k+1}$ in $\Omega_{k} \cap \Omega_{k+1}$ such that

$$
U_{j}(Z)=\left(1+\epsilon_{j}(Z)\right) \exp \left\{(-1)^{j} i Z\right\}
$$

and $\left|\epsilon_{j}(Z)\right|<C / \epsilon|Z|, j=k, k+1$ and by virtue of the range of $|Z|$, this is no greater than $1 / 4$. Now there exist $\alpha_{k}, \beta_{k} \in \mathbb{C}$ such that for $Z \in \Omega_{k} \cap \Omega_{k+1}$

$$
U(Z)=\alpha_{k} U_{k}(Z)+\beta_{k} U_{k+1}(Z) .
$$


If $\alpha_{k} \beta_{k}=0$, it is easily seen that $U$ has no zeros in $\Omega_{k} \cap \Omega_{k+1}$. If $\alpha_{k} \beta_{k} \neq 0$ and $U$ has a zero $Z_{0}$ in $\Omega_{k} \cap \Omega_{k+1}$, then since

$$
\alpha_{k}\left(1+\epsilon_{k}\left(Z_{0}\right)\right) \exp \left\{2(-1)^{k} i Z_{0}\right\}+\beta_{k}\left(1+\epsilon_{k+1}\left(Z_{0}\right)\right)=0,
$$

we have

$$
Z_{0}=\frac{(-1)^{k}}{2}\left[(2 l+1) \pi+i \log \frac{\alpha_{k}}{\beta_{k}}+i \log \left(\frac{1+\epsilon_{k}\left(Z_{0}\right)}{1+\epsilon_{k+1}\left(Z_{0}\right)}\right)\right],
$$

where $l \in Z$ and the arguments of $\alpha_{k} / \beta_{k}$ and $\left(1+\epsilon_{k}\left(Z_{0}\right)\right) /\left(1+\epsilon_{k+1}\left(Z_{0}\right)\right)$ are in $[0,2 \pi]$. Letting $\gamma_{k}=\log \left(\alpha_{k} / \beta_{k}\right)$ and $\lambda_{k, l}=i \log \left(\left(1+\epsilon_{k}\left(Z_{0}\right)\right) /\left(1+\epsilon_{k+1}\left(Z_{0}\right)\right)\right)$, we obtain (2.4). Moreover, by Lemma 3, and the fact that $R \geq 4 \sqrt{2} \pi C$, we have

$$
|\log | \frac{1+\epsilon_{k}\left(Z_{0}\right)}{1+\epsilon_{k+1}\left(Z_{0}\right)}||<\frac{4 C}{\epsilon\left|Z_{0}\right|}<\frac{4 C \sin \pi \epsilon}{\epsilon R}<\frac{4 \pi C}{R}
$$

and

$$
\left|\frac{1+\epsilon_{k}\left(Z_{0}\right)}{1+\epsilon_{k+1}\left(Z_{0}\right)}-1\right|<\frac{4 \pi C}{R} \leq \frac{1}{\sqrt{2}} .
$$

Denoting $\arg \left(\left(1+\epsilon_{k}\left(Z_{0}\right)\right) /\left(1+\epsilon_{k+1}\left(Z_{0}\right)\right)\right)$ by $\theta$, we consequently have that $|\sin \theta|<$ $4 \pi C / R,|\theta| \leq \pi / 4$, and $|\sin \theta|>|\theta| / 2$. Hence

$$
\left|\arg \left(\frac{1+\epsilon_{k}\left(Z_{0}\right)}{1+\epsilon_{k+1}\left(Z_{0}\right)}\right)\right|=|\theta|<2|\sin \theta|<8 \pi C / R .
$$

This and (2.6) imply (2.5).

\section{Proof of Theorem}

Since the exceptional set in Lemma 1 has finite logarithmic measure, applying Lemma 1 and Lemma 2 to the entire function $A(z)$, we see easily that there exists a sequence $\left\{\left[r_{n}^{\prime}, s_{n}^{\prime}\right]\right\}_{n \in \mathscr{G}}$ of closed intervals satisfying all the conclusions of Lemma 2 such that whenever $r=|z| \in \bigcup_{n \in \mathscr{G}}\left[r_{n}^{\prime}, s_{n}^{\prime}\right]$ and $r \rightarrow \infty$ we have

$$
\begin{aligned}
A(z) & =a_{N(r)}\left(1+\delta_{1}(z)\right) z^{N(r)}, \\
M\left(r, A^{(j)}\right) & =(1+o(1))(N(r) / r)^{j} M(r, A), \quad j=1,2
\end{aligned}
$$

and

$$
N(r) \equiv n, \quad r \in\left[r_{n}^{\prime}, s_{n}^{\prime}\right], \quad n \in \mathscr{G},
$$

where $\delta_{1}(z)$ satisfies

$$
\left|\delta_{1}(z)\right|<\exp \left\{-(\log |z|)^{\eta}\right\}, \quad 0<\eta<2-p .
$$


Moreover, without loss of generality, we can assume that

$$
\lim _{n \rightarrow \infty} s_{n}^{\prime} / r_{n}^{\prime}=\infty
$$

Now for sufficiently large $n \in \mathscr{G}$, we consider the following transform in each

$$
\mathscr{A}_{n}=\left\{z: r_{n}^{\prime} \leq|z| \leq s_{n}^{\prime},-\infty<\arg Z<\infty\right\},
$$

a closed region on the Riemann surface of $\log z$. For convenience, we write

$$
\mathscr{A}=\left\{z: s \leq|z| \leq s^{\prime},-\infty<\arg z<\infty\right\}
$$

for any $\mathscr{A}_{n}$ above. Noting (3.3), consider the transform

$$
Z=\int_{s}^{z} A(t)^{1 / 2} d t+2 /(n+2) a_{n}^{1 / 2} s^{(n+2) / 2}, \quad z \in \mathscr{A},
$$

where the integral is evidently independent of the path, which remains in $\mathscr{A}$. As in [3, Chap.7], we define $W$ as in (1.6). Then if $w$ is a solution of (1.1), $W$ solves (1.4), and $F(Z)$ is given by (1.5). Moreover, (3.1) and (3.3) imply that

$$
Z=\frac{2}{n+2} a_{n}^{1 / 2} z^{(n+2) / 2}+a_{n}^{1 / 2} \int_{s}^{z} t^{n / 2} \delta_{2}(t) d t, \quad z \in \mathscr{A},
$$

where $\delta_{2}(z)$ satisfies $\left|\delta_{2}(z)\right|<\exp \left\{-(\log |z|)^{\eta}\right\}$ in $\mathscr{A}$. So, if $-3 \pi \leq \arg z \leq 3 \pi$, choose the path of integration in (3.6) to be the straight line segment from $s$ to $|z|$, followed by the arc of $|t|=|z|$ from $|z|$ to $z$ in $\mathscr{A}$. Letting $d \tau$ denote arc-length on the path, we have

$$
\begin{aligned}
\left|Z-\frac{2}{n+2} a_{n}^{1 / 2} z^{(n+2) / 2}\right| & \leq\left|a_{n}\right|^{1 / 2} \int_{s}^{z}|t|^{n / 2} \exp \left\{-(\log |t|)^{\eta}\right\} d \tau \\
& =\left|a_{n}\right|^{1 / 2}\left\{\int_{s}^{z z \mid} x^{n / 2} \exp \left\{-(\log x)^{\eta}\right\} d x+\int_{|z|}^{z}|z|^{n / 2} \exp \left\{-(\log |z|)^{\eta}\right\} d \tau\right\} \\
& \leq\left|a_{n}\right|^{1 / 2}\left\{\exp \left\{-(\log s)^{\eta}\right\} \cdot|z|^{(n+2) / 2}+3 \pi \exp \left\{-(\log |z|)^{\eta}\right\} \cdot|z|^{(n+2) / 2}\right\} \\
& \leq(1+3 \pi)\left|a_{n}\right|^{1 / 2}|z|^{(n+2) / 2} \exp \left\{-(\log s)^{\eta}\right\} .
\end{aligned}
$$

According to Lemma 2,

$$
N(r) \equiv n<(\log r)^{q-1}
$$

if $s \leq r \leq s^{\prime}$, where $p<q<2-\eta$. Thus (3.7) implies that

$$
Z=\left(1+\delta_{3}(z)\right) \frac{2}{n+2} a_{n}^{1 / 2} z^{(n+2) / 2},
$$


where $\delta_{3}(z)$ satisfies

$$
\left|\delta_{3}(z)\right|<\exp \left\{-(\log s)^{\eta} / 2\right\}
$$

for all $z \in \mathscr{A}$ with $-3 \pi \leq \arg z \leq 3 \pi$. Therefore the transform (3.5) maps

$$
\left\{z: s \leq|z| \leq s^{\prime},-3 \pi \leq \arg z \leq 3 \pi\right\}
$$

one-to-one onto a set containing

$$
\mathscr{B}=\left\{Z: S \leq|Z| \leq S^{\prime}, \frac{2 \alpha_{n}-5(n+2) \pi}{4} \leq \arg Z \leq \frac{2 \alpha_{n}+5(n+2) \pi}{4}\right\},
$$

where $S=3 \sqrt{\left|a_{n}\right|} s^{(n+2) / 2} /(n+2), S^{\prime}=\sqrt{\left|a_{n}\right|} s^{(n+2) / 2} /(n+2)$ and $\alpha_{n}=\arg a_{n}$. A routine calculation involving (3.1), (3.2), (3.3), (1.5) and (3.9), gives for $Z \in \mathscr{B}$ that

$$
|F(Z)|<C /|Z|^{2} .
$$

Certainly Lemma 2 implies that $A(z)$ has no zeros in $\mathscr{A}$ so that $F(Z)$ is analytic in $\mathscr{B}$. By (3.4) we can assume that $S^{\prime}>S(\sin \pi \epsilon)^{-1}$. Then $F(Z)$ satisfies the conditions of Lemma 3. Hence (1.4) has a solution $U_{k}(Z)$ in

$$
\mathscr{C}_{k}=\mathscr{B} \cap\left\{Z: S(\sin \pi \epsilon)^{-1} \leq|Z| \leq S^{\prime},(k-1+\epsilon) \pi \leq \arg Z \leq(k+2-\epsilon) \pi\right\}
$$

such that

$$
U_{k}(Z)=\left(1+\epsilon_{k}(Z)\right) \exp \left\{(-1)^{k} i Z\right\}
$$

and

$$
\left|\epsilon_{k}(Z)\right|<C / \epsilon|Z|
$$

in $\mathscr{C}_{k}$, where $k \in \mathbb{Z}$. We can also assume that $n \in \mathscr{G}$ is sufficiently large so that $S>C^{2} \epsilon^{-2}$. Then we have

$$
\left|\epsilon_{k}(Z)\right|<1 /|Z|^{1 / 2} .
$$

Further let $n \in \mathscr{G}$ be large enough so that $e^{n+2}>(\sin \pi \epsilon)^{-1}$ and let

$$
\mathscr{D}=\left\{z: 3 e^{2} s \leq|z| \leq s^{\prime} / 3 e^{2},-2 \pi \leq \arg z \leq 2 \pi\right\} .
$$

Then from (3.9) we know that the transform (3.5) maps $\mathscr{D}$ one-to-one onto a subset of $\mathscr{B} \cap\left\{Z: S(\sin \pi \epsilon)^{-1} \leq|Z| \leq S^{\prime}\right\}$. Hence the equation (1.1) has a solution $u_{k}$ in the intersection of $\mathscr{D}$ and the preimage of $\mathscr{C}_{k}$ which contains

$$
\mathscr{E}_{k}(\epsilon)=\mathscr{D} \cap\left\{z: \frac{-\alpha_{n}+2(k-1) \pi}{n+2}+\epsilon \leq \arg z \leq \frac{-\alpha_{n}+2(k+2) \pi}{n+2}-\epsilon\right\}
$$


where $\alpha_{n}=\arg a_{n}$ and $\epsilon>0$. By (3.1), (3.3), (1.6), (3.9), (3.10), (3.11) and (3.12), for $z \in \mathscr{E}_{k}(\epsilon)$,

$$
u_{k}(z)=(1+o(1))\left(a_{n} z^{n}\right)^{-1 / 4} \exp \left\{\frac{2}{n+2} \sqrt{\left|a_{n}\right|} e^{i \alpha_{n} / 2}(-1)^{k} i z^{(n+2) / 2}(1+o(1))\right\} .
$$

Since $\mathscr{A}$ denotes any $\mathscr{A}_{n}$ with sufficiently large $n \in \mathscr{G}$, if we let

$$
r_{n}=3 e^{2} r_{n}^{\prime}, \quad s_{n}=s_{n}^{\prime} / 3 e^{2}
$$

for sufficiently large $n \in \mathscr{G}$, we have actually proved (1.8) in $\Omega_{n, k}(\epsilon)$ (provided we redefine $\mathscr{G}$ to contain only sufficiently large $n$ ). Moreover, noting (3.13) and (3.8) and using the definition of the logarithmic density, we can easily see that the logarithmic density of $\bigcup_{n \in \mathscr{G}}\left[\left(r_{n}^{\prime}, r_{n}\right) \cup\left(s_{n}, s_{n}^{\prime}\right)\right]$ is zero.

Finally, if $u$ is a solution of (1.1), then by Lemma 4 and (3.9) for $n \in \mathscr{G}$ either $u$ has no zeros in $\Omega_{n, k}(\epsilon) \cap \Omega_{n, k+1}(\epsilon)$ or the zeros of $u$ satisfy (1.9). We obtain (1.10) from (3.10) and (1.11) from (2.5), Lemma 2 and the fact that $a_{N} r_{N}^{N}$ increases to infinity with $n[1, \mathrm{p} .318]$.

\section{References}

[1] W. K. Hayman, 'The local growth of power series: a survey of the Wiman-Valiron method', Canad. Math. Bull. 17 (1974), 317-358.

[2] E. Hille, 'Oscillation theorems in the complex domain' Trans. Amer. Math. Soc. 23 (1922), 350385.

[3] __ Lectures on ordinary differential equations (Addison-Wesley, London, 1969).

[4] J. K. Langley, 'Proof of a conjecture of Hayman concerning $f$ and $f$ "', preprint.

Department of Mathematics

Virginia Polytechnic Institute and State University

Blacksburg, VA 24061

USA

e-mail: rossi@math.vt.edu 